\title{
Medicine in a Neurocentric World: About the Explanatory Power of Neuroscientific Models in Medical Research and Practice
}

\author{
Lara Huber • Lara K. Kutschenko
}

Received: 5 November 2009/Accepted: 7 December 2009/Published online: 29 December 2009

(C) Springer Science+Business Media B.V. 2009

\begin{abstract}
Explanatory models in the neurosciences are seen to have a growing impact on medical regimes of research and practice. At this stage, new diagnostic tools and therapeutic interventions have altered the shape of clinical disciplines such as neurology and psychiatry. This development is driven by specific modes of operationalisation, which immediately call for new standardisation procedures. In the long run, neuroscientific models are expected to follow the reconceptualisation of classification systems, hence contributing to a change in the understanding of somatic and mental disease entities. Medicine in a neurocentric world is dedicated to the analysis of how current regimes of medical research and practice are influenced by neuroscientific approaches to brain and mind. At least three major issues could be identified: how particular explanatory models have evolved, how they are introduced to the medical field and how they are transforming medical research and practice. Anthropological, ethical and cultural challenges arising from the alignment of neuroscience and medicine are addressed. Historical studies concerning the methodological preconditions of neuroscientific research on the one hand and strategies of intervening in the brain
\end{abstract}

L. Huber $(\bowtie) \cdot$ L. K. Kutschenko

Institute for History, Philosophy and Ethics of Medicine, University Medical Center of the Johannes Gutenberg University Mainz, Am Pulverturm 13, 55131 Mainz, Germany

e-mail: huberl@uni-mainz.de and mind in clinical practice on the other are rounding off this themed issue.

Keywords Neurology · Psychiatry · Neuroscience $\cdot$ Explanatory models . Experimental regimes - Functionality · Exceptionality · Philosophy of medicine . Ethics

"Given the complementary strengths of neuroimaging and patient studies, we predict that the most successful cognitive neuroscience research programs of the twenty-first century will be those that combine the two approaches." (Farah and Feinberg 2006, p. 17).

Explanatory models in the neurosciences are seen to have a growing impact on medical regimes of research and practice. At this stage, new diagnostic tools (e.g. neuroimaging) and therapeutic interventions (e.g. deep-brain stimulation) have altered the shape of clinical disciplines such as neurology and psychiatry. This development is driven by specific modes of operationalisation, which immediately call for new standardisation procedures (e.g. brain atlases). In the long run, neuroscientific models are expected to follow the reconceptualisation of classification systems (e.g. DSM), hence contributing to a change in the understanding of somatic and mental disease entities. 
As a starting point for an analysis in medicine studies, it is crucial to evaluate why models that arise out of neuroscientific research are attributed a specific explanatory power. One answer to this question, and a pragmatic one at that, would be (1) that from today's perspective the heuristics of these models allow for a variety of applications ('functionality of neuroscientific models' thesis). Another answer could be (2) that these models directly benefit from the very fact that the neurosciences are being ascribed a specific explanatory power ('exceptionality of neuroscientific models' thesis).

1. Functionality of neuroscientific models: This approach asks in how far the high performability of explanatory models in the neurosciences strengthens their presumed explanatory power. With regard to the evaluation of their epistemic validity, ${ }^{1}$ first of all, one has to assess how specific experimental strategies underlying these models perform. Drawing upon the instrumental preconditions of experimental strategies, currently the epistemic reach of neuroscientific models is under debate: Particularly, this is as much due to methodological shortfalls of highly technicised laboratory settings in cognitive neuroscience (Bechtel 2002; Hardcastle and Stewart 2005) as to epistemological challenges of converging technologies (e.g. NBIC: Nano-Bio-Info-Cogno). Additionally, the instrumental preconditions of experimental approaches are critically reflected with regard to their historicity, given that they are contributing to a reconfiguration of standards of scientific objectivity (Galison 1998).

2. Exceptionality of neuroscientific models: In principle, this hypothesis is based on the assumption that in comparison with other biomedical information, neural information is of a significantly different quality. According to neuroessentialism (Crick and Clarck 1994; Roskies 2002), neurons are regarded as the material basis of mental phenomena and, therefore, they are assigned a specific informational value concerning fundamental anthropological phenomena such as

\footnotetext{
${ }^{1}$ Evaluating their epistemic validity means to assess both their performance within a specific experimental setting (internal validity: models are designed and controlled to establish just the results they claim) and their applicability to other settings (external validity).
}

personal identity. From an epistemological point of view, two questions arise: The dichotomic understanding of information ${ }^{2}$ with a structuralquantitative approach ( $\left.i^{\text {quantitative }}\right)$ on the one side and a semantic-qualitative one ( $\left.i^{\text {qualitative }}\right)$ on the other raises the question whether and how the transition from ( $\left.\mathrm{i}^{\text {quantitative }}\right)$ to (i $\left.\mathrm{i}^{\text {qualitative }}\right)$ could be conceptualised within the realm of the philosophy of neuroscience. Additionally, it is noticeable that epistemic strategies for clarifying the personal relatedness of neural information are still unavailable, and this absence is considered as a key problem for neuroexceptionalism (Huber 2009a). Nevertheless, taking the genetic exceptionalism discourse $^{3}$ as a precedent, the sensitive nature of neural information, in recent years, has triggered debates on how neuroscience poses unique (neuro) ethical challenges (Illes and Racine 2005; Wachbroit 2008).

With regard to Medicine in a neurocentric world, at least three major themes could be identified that pave the road for transdisciplinary studies. Different methodological approaches in this field are explicitly or implicitly linked to epistemological questions. Also, the historicity and the normativity of epistemological challenges have to be taken into account (cf. Fleck 1980). Hence, the aim of this issue is to analyse (a) how particular explanatory models have evolved, (b) how they are introduced to the medical field and (c) how they are shaping medical research and practice.

a. To examine how neuroscientific models have evolved is, first, to identify essential components of experimental design and, secondly, to analyse these methodological features in relation to their specific epistemic reach: the first point addresses strategies of operational and instrumental knowhow (Bechtel and Stufflebeam 2001). The latter stresses issues of theoretical structure and scientific modelling (Hardcastle and Stewart 2001).

\footnotetext{
${ }^{2}$ See, for the polysemantic character of information, Floridi (2005).

3 A current analysis of the genetic exceptionalism discourse can be found in Ilkilic (2009); for a discussion of how molecular biology on the one side and the neurosciences on the other afford penetrating insights into health conditions see, for example, Green (2006).
} 
With regard to Medicine in a neurocentric world, one might ask how experimental regimes have contributed to a stabilisation of theoretical underpinnings (e.g. "neuro-doctrin").

b. To analyse how neuroscientific models are introduced to the medical field, in particular, is to deconstruct in how far the expected functionality of certain models is based on their specific explanatory power or if it merely comes along with the introduction of specific "biomedical platforms" (Keating and Cambrosio 2003). It can be asked, for example, to what degree explanatory models are intertwined and, therefore, "packaged" with modes of operationalisation as well as technological preconditions (Fujimura 1982; Lenoir 1988).

c. The latter immediately affects the third question: To analyse how neuroscientific models have shaped medical research and practice is to pick out as a central theme the epistemic impact of their functionality. Drawing upon the pragmatic character of systems of classification (Bowker and Star 2000), this question focuses specifically on issues of integrating very different explanatory models into medical nomenclature. Given that neuroscientific approaches are currently just one resource for explanatory models, though an exceptionally potent one, the pressing issue remains to what degree these models are taking part in the (re)conceptualisation of nosological entities.

Against this background, the very epistemic goals of medical research have to be taken into account. Applicability could be regarded as an essential aim of medical research. Central to any discussion about how applicability can be achieved is to define to what degree processes of validation and standardisation (e.g. normative samples) contribute to it. From an epistemological point of view, various challenges arise out of methodological preconditions such as defining homogeneous patient groups or modelling human disease entities in animal-based research. In adjusting to these challenges, additional (pragmatic) tools (e.g. probability atlases) are often introduced. These can even influence experimental regimes themselves. When it comes to translating experimental data into clinical application, epistemological problems may even be reinforced (Paul 1998). As a matter of fact, the epistemic functionality of given strategies in medical research itself is challenging the ideal of clinical usefulness: The prerequisites of stabilising experimental data (e.g. homogeneity of study population) evoke the question in how far these data could be used in medical practice, given the heterogeneity of individual pathology.

Problems in medical practice arise from the explanatory power that is commonly associated with new experimental models evolving out of neuroscientific research. Furthermore, it is to be noticed that epistemological challenges in medical research often are accompanied by ethical challenges in medical practice (e.g. recruitment of patients as research subjects). For instance, this could be seen in the process of introducing new technologies that are expected to improve diagnostic know-how. Looking at current strategies of imaging in psychiatry, the diagnostic power of new technologies is still under debate (Huber 2009b). As a matter of fact, issues of visualising specific pathological entities (e.g. neuroimaging) for diagnostic purposes always go hand-inhand with the aim of therapeutic intervention (e.g. psychopharmacology). Thus, there is at least one lesson to be learned from the ethical assessment of genetic screening: In order to meet the aims of good medical practice, a thorough evaluation of the outcome (e.g. side effects of diagnostics) should always be weighed against the possibility of therapeutic interventions available for the individual patient (e.g. "therapeutic gap"). ${ }^{4}$ Of course, ethical assessment of the outcome is also an issue for therapeutic interventions, involving, for example, the expansion of therapeutic indications (e.g. neurosurgical interventions in psychiatry). In analysing the technicity of medical regimes, Henk Ten Have famously pointed out how the very tools of diagnostics and therapeutics "bring about new demarcations between what is medical and what is nonmedical" (ten Have 1995, 16).

In sum, medical practice not only responds to societal debates on health-related issues, but also

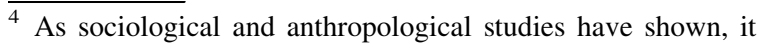
might also be an issue in this context to evaluate how medical practices are shaping an individual's self. On reconceptualisations (of individuals) as a consequence of the biological turn in psychiatry, see for example Dumit $(1997,2003)$, for a general discussion of "thinking of kinds of people as objects of scientific inquiry", see Hacking (2006, p. 2).
} 
impacts on what is defined as falling into the medical domain. This means, first, to reflect how disease entities co-evolve ${ }^{5}$ with societal conceptualisations of abnormal behaviour and how the availability of technological and/or psychopharmacological tools of intervention strengthens societal perceptions of cognitive and emotional deficits (e.g. hyperactivity). Hence, current debates on ethical and societal pitfalls of "technological fixes"6 (e.g. cognitive or mood enhancement) are concerned with the question whether certain challenges known to be based on societal grievances first and foremost could reasonably be dissolved with mere technological or pharmacological approaches. Against the background of practices of diagnostic expansion, so-called trends of "medicalisation" of certain societal groups (e.g. the elderly) are reported that might deeply affect an individual's life and physical condition (Conrad 2007).

Medicine in a neurocentric world is dedicated to the analysis of how current regimes of medical research and practice are influenced by neuroscientific approaches to brain and mind. ${ }^{7}$ Anthropological, ethical and cultural challenges at the interface of neuroscience and medicine are addressed. Historical studies concerning the methodological preconditions of neuroscientific research on the one hand and strategies of intervening in the brain and mind in clinical practice on the other are rounding off this themed issue.

Nicolas Kopp as a neurologist opens this themed issue with an in-depth review of the past and present of imaging technology in neurology. Bringing in his expertise as a practitioner, he addresses how new technological tools (e.g. MRI) are shaping clinical research and practice in neurology. Kopp's analysis starts with an introduction to current strategies of imaging in neurology. In referring to changes in brain morphology and function that are considered to carry pathological values, he discusses major advantages

\footnotetext{
5 See, for example, the debate on "co-construction": Latour (1997); Jasanoff (2006). For a historical study addressing this issue with regard to concepts of de- and regeneration in the German morphological neurosciences, see Stahnisch (2009).

${ }^{6}$ For the introduction of this very notion, see Rosner (2004).

${ }^{7}$ In a way, Stephen Rose's critical note on "Ethics in a neurocentric world" (Rose 2007) has inspired our call for papers.
}

for diagnostic and therapeutic purposes that arise from these imaging modalities. In addition, Kopp also critically reflects upon how expected outcomes are in conflict with risks for patients linked to imaging technologies as well as with expectations of patients impressed by the visual appeal of technologically produced images.

New strategies of visualising brain morphology and function are more and more influencing not only lay expectations, but also contributing to a reductive scientific perspective on neuroscientific research as being merely imaging based. In particular, current cognitive neuroscience is equated with elaborated modes of representation such as functional magnetic resonance imaging (fMRI). Cornelius Borck invites the reader on a memorable journey to early neuroscientific dreams of the "transparent mind and brain". 8 His historical analysis of physiological recordings of brain function (e.g. EEG) stresses epistemological challenges of reductionist concepts that relate today's neuroscience to its past history. Here, Borck provides a starting point for further philosophical studies focussing on an epistemic evaluation of protocols in neuroscientific research encompassing current paradigms in medical research and practice.

At the epistemic intersection of medical research and clinical practice, Jean-Gaël Barbara deconstructs the origin of experimental and scientific models of pathogeny in diseases of the nervous system in nineteenth-century Paris. Drawing upon the complexity of disease entities, Barbara analyses how convergences between clinical observations, pathological data and theoretical underpinnings of experimental modelling were established. Also, he stresses the introduction of new transdisciplinary explanatory systems, so-called "search engines of pathogenies" in this field of research that were strengthened by experimental and clinical investigations, especially.

Stabilising clinical observation and validating medical intervention could be regarded as key figures of medical research. Fundamental challenges arise out of the specific epistemological setting of this field of research. One problem is the empirical underdetermination of medical practice, which, as several studies have shown, may lead to an expansion of

\footnotetext{
8 For the ideal of the "transparent body" see, for example, van Dijck (2005).
} 
regimes of research into medical practice. ${ }^{9}$ This immediately affects the very relationship of physicians to their patients, as Marietta Meier illustrates in the context of her historical study on "psychosurgical" practice in a Zurich mental asylum after World War II. Here, she critically reflects medical practices of socially adjusting patients that are regarded as mentally ill. Meier's study discusses how prefrontal lobotomy as a surgical strategy of intervening into brain and mind was based on the emergence of a new concept of individual functioning in society. In the presence of these and other societal and ethical challenges, Jenell Johnson explores in how far current therapeutic options in the United States are shaped by a "collective memory" of medical malpractices associated with psychosurgical interventions in the 1950 s to 1970 s. ${ }^{10}$ Drawing upon public perceptions of biomedicine and surgical practice, Johnson's study is mainly focused on an evaluation of the rhetoric of American medicine and popular culture. Here, he discusses how rhetorical strategies that bypass notions evoking negative associations have impacted on the very dialogue between physicians, patients and their relatives, for example, in the decision-making process regarding deep-brain stimulation (DBS) as a treatment option.

Against the background of current clinical approaches of intervening into brain and mind on the one side and the societal debate on cognitive enhancement on the other, Dominik Groß discusses in how far medical ethics should put nonpharmacological strategies of enhancement on its agenda. $\mathrm{He}$ analyses key arguments of this debate, e.g. the specific quality of "brain engineering" (viz. invasiveness). Furthermore, Groß stresses the problem of anticipating future technological developments and their impact on (mis)judging trends in medical ethics. Taking these and other debates in bioethics as a starting point, Cordula Brand poses the question whether "personal identity" as a theoretical concept could be applied to the normative evaluation of

\footnotetext{
${ }^{9}$ For an evaluation of the experimental turn in medicine from an epistemological point of view, see Bernard (2008); Canguilhem (1965); Fleck (1980). For a historical study, see for example, Gradmann (2005).

10 For the differentiation between "leucotomy" and "lobotomy" as well as for other surgical strategies of intervening in the brain with psychiatric indication, see Meier (this issue); Johnson (this issue).
}

health-related issues. Investigating key features of this concept, she sketches given theoretical problems of "personal identity" (e.g. human persistence), introducing an alternative interpretation. Also, Brand discusses the functionality of her concept with regard to specific challenges in medical practice, especially concerning DBS.

\section{Further Research}

The papers in Medicine in a neurocentric world illustrate that various disciplines and scientific communities are engaged in debates over medical research and practice that are influenced by neuroscientific approaches to brain and mind. Accordingly, this issue includes quite different methodological strategies and scientific perspectives between neuroscience and medicine. Against this background, there are at least three themes that emerge out of the transdisciplinary setting of Medicine in neurocentric world: (1) the inherent normativity of explanatory models that arise out of neuroscientific approaches; (2) the expansion of regimes of research into medical practice; (3) medicine-based approaches to cognitive neuroscience.

1. The inherent normativity of scientific strategies of comparing individual data to standardised statistical values led the scientific community to bypassing the notion of "normative samples" or "normal atlases", while introducing so-called "reference values" in the 1980s (Büttner 1997). Nevertheless, it is worth noting that procedures of normalising, ${ }^{11}$ e.g. atlasing brain variability (Toga et al. 2001), immediately affect the societal perception of normal functioning. Drawing upon studies in the philosophy of medicine (Canguilhem 1966) as much as on studies in social history (Foucault 1975, 1999), two directions of future research in medicine studies could be, (a) to evaluate whether the explanatory power

\footnotetext{
11 Alongside specific scientific practices of comparing individual data to standardised data sets, normalising could be understood as an issue of "procedural validity" (verfahrenstechnologische Normalisierung). In this regard, as an innerscientific regime, it has to be differentiated against issues of "societal reliability" (Normalisierung als Veralltäglichung). For an introduction to this differentiation, see Huber (2009c).
} 
of neuroscientific models gains or loses from the fuzzy character of the very concept of normality in medicine; (b) to develop new strategies of responding to the scientific discourse on practices of normalisation, especially considering effects on societal perception of normal functioning (e.g. against the background of an ethical assessment addressing issues of "desirability").

2. The expansion of regimes of research into medical practice could be regarded as a major challenge for (a) epistemology of medicine on the one side and (b) ethics in biomedicine on the other: future research may critically reflect (a) current methodological transformations of research paradigms due to the specific demands of patient-based approaches in clinics (e.g. research on prodromal stages of mental disorders), as well as (b) effects on patients (and their relatives) of being offered specific diagnostic and therapeutic options merely in virtue of participating in a clinical trial.

3. As a matter of fact, current challenges of methodological transformations in this field are due to the increasing use of biomedical platforms, imaging technologies as much as of neuropsychological tests and protocols. To introduce new experimental models arising out of neuroscientific research into various fields of medical practice implies not only the reshaping of clinical gold standards, but also a transformation of the self-perception of clinical disciplines more and more engaging in neuroscientific research themselves. ${ }^{12}$ Taking medicine-based approaches to cognitive neuroscience as a starting point, future research might focus on the epistemic validity of the presumption that clinical studies may explain (normal) cognitive functioning-given that studies on a patientspecific basis at least suggest that individual pathology may result in or just coincide with morphological and or functional changes-considering brain plasticity or side effects of ataractics (e.g. Barch 2006).

\footnotetext{
12 In recent years, several conferences have focused on the specific contribution of medical research to cognitive neuroscience (Tatagiba et al. 2007; WFSBP 2009). Also, more and more scientific papers are dealing with this issue (Farah and Feinberg 2006; Broome and Bortolotti 2009).
}

The possibilities for future work appear unlimited. Still, our hope is that this themed issue on Medicine in a neurocentric world will stimulate an interest in how explanatory models from the neurosciences are set up and reflected within various medical practices. Drawing upon the epistemological, ethical and societal consequences of the "neurocentricity" of medicine, the goal of this issue is to clarify at least some of the challenges that arise from the alignment of neuroscience and medicine, hence serving as a useful reference for future research in Medicine Studies.

\section{References}

Barch, Deanna M. 2006. What can research on schizophrenia tell us about the cognitive neuroscience of working memory? Neuroscience 139: 73-84.

Bechtel, William. 2002. Aligning multiple research techniques in cognitive neuroscience: Why is it important? Philosophy of Science 69: S48-S58.

Bechtel, William, and Robert S. Stufflebeam. 2001. Epistemic issues in procuring evidence about the brain: The importance of research instruments and techniques". In Philosophy and the neurosciences. A reader, ed. William Bechtel, et al., 55-81. Oxford: Blackwell Publishers.

Bernard, Claude. 2008. Introduction à l'étude de la médicine expérimentale (Paris 1865). ed. Fabrice Gzil. Paris: Librairie Générale Française.

Bowker, Geoffrey C., and Susan Leigh Star. 2000. Sorting things out. Classification and its consequences. London: The MIT Press.

Broome, Matthew, and Lisa Bortolotti. 2009. Psychiatry as cognitive neuroscience. Philosophical perspectives. Oxford: Oxford University Press.

Büttner, Johannes. 1997. Die Herausbildung des NormalwertKonzeptes im Zusammenhang mit quantitativen diagnostischen Untersuchungen der Medizin. In Normierung der Gesundheit. Messende Verfahren der Medizin als kulturelle Praktik um 1900, ed. Volker Hess, 17-32. Husum: Matthiesen Verlag.

Canguilhem, Georges. 1965. La connaissance de la vie. Paris: J. Vrin.

Canguilhem, Georges. 1966. Le normal et le pathologique. Paris: Presses Universitaires de France.

Conrad, Peter. 2007. The medicalization of society. On the transformation of human conditions into treatable disorders. Baltimore: Johns Hopkins University Press.

Crick, Francis, and J. Clarck. 1994. The astonishing hypothesis. Journal of Consciousness Studies 1 (1): 10-16.

Dumit, Joseph. 1997. A digital image of the category of the person. In Cyborgs \& Citadels. Anthropological interventions in emerging sciences and technologies, ed. Gary Lee Downey, and Joseph Dumit, 83-102. Santa Fe: School of American Research Press.

Dumit, Joseph. 2003. Is it me or my brain? Depression and neuroscientific facts. Journal of Medical Humanities 24 (1-2): 35-47. 
Farah, Martha J., and Todd E. Feinberg. 2006. Patient-based approaches to cognitive neuroscience, 2nd ed. Cambridge: The MIT Press.

Fleck, Ludwik. 1980. Entstehung und Entwicklung einer wissenschaftlichen Tatsache. In Einführung in die Lehre vom Denkstil und Denkkollektiv, ed. Lothar Schäfer, and Thomas Schnelle. Frankfurt: Suhrkamp Verlag.

Floridi, Luciano. 2005. Semantic conceptions of information. In Stanford encyclopedia of philosophy. http://plato.stanford. edu/entries/information-semantic/ Accessed 19 October 2009.

Foucault, Michel. 1975. Surveiller et punir. Naissance de la prison. Paris: Gallimard.

Foucault, Michel. 1999. Les anormales. Cours au collège de France. Paris: Seuil.

Fujimura, Joan H. 1982. Crafting science: Standardized packages, boundary objects, and "translation". In Science as practice and culture, ed. Andrew Pickering, 168-211. London: University of Chicago Press.

Galison, Peter. 1998. Judgment against objectivity. In Picturing science, producing art, ed. Caroline A. Jones, and Peter Galison, 327-359. London: Routledge.

Gradmann, Christoph. 2005. Krankheit im Labor. Robert Koch und die medizinische Bakteriologie. Göttingen: Wallstein Verlag.

Green, Ronald M. 2006. From genome to brainome: charting the lessons learned. In Neuroethics. Defining the issues in theory, practice, and policy, ed. Judy Illes, 105-121. Oxford: Oxford University Press.

Hacking, Ian. 2006. Kinds of people: Moving targets. In: The tenth British academy lecture. London: The British Academy.

http://www.britac.ac.uk:80/pubs/src/britacad06/index.cfm . Accessed 29 October 2009.

Hardcastle, Valerie Gray, and C. Matthew Stewart. 2001. Theory structure in the neurosciences. In Theory and method in the neurosciences, ed. Peter K. Machamer, Rick Grush, and Peter McLaughlin, 30-44. Pittsburgh: University of Pittsburgh Press.

Hardcastle, Valerie Gray, and C. Matthew Stewart. 2005. Localization in the brain and other illusions. In Cognition and the brain. The philosophy and neuroscience movement, ed. Andrew Brook, and Kathleen Akins, 27-39. Cambridge: Cambridge University Press.

Huber, Lara. 2009a. Exzeptionalismus "revisited" oder: Von der Naturalisierung durch Technisierung. In Das technisierte Gehirn. Neurotechnologien als Herausforderung für Ethik und Anthropologie, ed. Oliver Müller, Jens Clausen, and Giovanni Maio, 118-136. Paderborn: Mentis Verlag.

Huber, Lara. 2009b. Imaging the brain-"visualising pathological entities"? Searching for reliable protocols within psychiatry and their impact on the understanding of psychiatric diseases. Poiesis \& Praxis 6 (1/2): 27-41.

Huber, Lara. 2009c. Operationalisierung-StandardisierungNormalisierung: Die Produktion und Visualisierung von Daten in der kognitiven Neurowissenschaft. In Erkenntnis und Kritik. Zeitgenössische Positionen, ed. Devi Dumbadze, et al., 167-191. Bielefeld: transcript Verlag.

Ilkilic, Ilhan. 2009. Coming to grips with genetic exceptionalism: Roots and reach of an explanatory model. Medicine Studies 1 (2): 131-142.

Illes, Judy, and Eric Racine. 2005. Imaging or imagining? A neuroethics challenge informed by genetics. The American Journal of Bioethics 5 (2): 5-18.

Jasanoff, Sheila. 2006. States of knowledge. The co-production of science and social order. London: Routledge.

Keating, Peter, and Alberto Cambrosio. 2003. Biomedical platforms. Realigning the normal and the pathological in latetwentieth-century medicine. Cambridge: The MIT Press.

Latour, Bruno. 1997. Nous n'avons jamais été modernes: Essai d'anthropologie symétrique. Paris: Éditions la Découverte \& Syros.

Lenoir, Timothy. 1988. Practice, reason, context: The dialogue between theory and experiment. Science in Context 2 (1): 3-22.

Paul, Norbert. 1998. Incurable suffering form the "hiatus theoreticus"? Some epistemological problems in modern medicine and the clinical relevance of philosophy of medicine. Theoretical Medicine and Bioethics 19: 229-251.

Rose, Stephen. 2007. Ethics in a neurocentric world. In Defining right and wrong in brain sciences. Essential readings in neuroethics, ed. Walter Glannon, 369-377. New York: Dana Press.

Roskies, Adina. 2002. Neuroethics for the new millennium. Neuron 35: 21-23.

Rosner, Lisa. 2004. The technological fix. How people use technology to create and solve problems. New York: Routledge.

Stahnisch, Frank W. 2009. Transforming the lab: technological and societal concerns in the pursuit of de- and regeneration in the German morphological neurosciences, 19101930. Medicine Studies 1 (1): 41-54.

Tatagiba, Marcos et al. 2007. Combining neuroscience with neurosurgery. Proceedings of the 1 st international symposium on cognitive neurosurgery. 17-20 March 2007. Castle of Weitenburg, Germany. Kirchentellinsfurt: Knirsch Verlag.

Ten Have, Henk. 1995. Medical technology assessment and ethics ambivalent relations. Hasting Center Report 25: 13-19.

Toga, Arthur W., et al. 2001. Probabilistic approaches for atlasing normal and disease-specific brain variability. Anatomy and Embryology 204: 267-282.

Van Dijck, José. 2005. The transparent body. A cultural analysis of medical imaging. London: University of Washington Press.

Wachbroit, Robert. 2008. The prospects for neuro-exceptionalism: Transparent lies, naked minds. The American Journal of Bioethics 8 (1): 3-8.

World Federation of Societies of Biological Psychiatry (WFSBP). 2009. Abstracts of the 9th world congress of biological psychiatry, 28 June-02 July 2009, Paris/France, WFSBP. doi:10.3284/wfsbp.2009.1. 